\title{
Educación en valores y educación moral: un ámbito de diseño y construcción curricular para el profesorado
}

\section{Miquel Martínez}

\section{$R x$}

En este artículo se presenta la Educación en valores y la Educación Moral como un ámbito ótimo para la práctica del profesorado en la construcción de actividades de caracter transversal. Se analizan algunos de los problemas y cuestiones a tener en cuenta ante toda innovación pedagógica y se sugieren pautas para abordar la propuesta que se formula de forma eficaz. Se trata, una vez más, de relacionar teoría y práctica de forma que sea posible incrementar la eficacia de la acción pedagógica y a la vez el rigor y la sistematicidad teórica.

La reforma del sistema educativo, las orientaciones y diseños curriculares que deberán concretarse en el marco del aula y en el espacio de los centros educativos, plantean un importante reto a los profesionales de la educación. La reforma plantea como mínimo un reto que debemos caracterizar como complejo y que obliga al profesorado a reflexionar sobre cuál es su función en el aula, en qué grado debe integrarse necesariamente en un trabajo en equipo y sobre el tipo de adecuación entre su forma de hacer en el aula y el grado de transversalidad que caracteriza el modelo curricular derivado de la reforma.

Probablemente, no sea el momento más idónea para plantear nuevas demandas al profesorado, pero es obvio que el éxito de la reforma depende en gran medida de un cambio notable en la mentalidad del profesorado y en la forma de abordar sus funciones pedagógicas.

Existen formas más eficaces que otras de abordar el trabajo diario en el aula y las innovaciones pedagógicas que los diseños curriculares pueden conllevar. Y existen, por supuesto, formas de dudosa eficacia que, además, pueden contribuir al desánimo y a la pérdida de la ilusión profesional. 
Los planes de formación permanente del profesorado pueden contribuir, sin duda, a la mejora de la calidad de la educación y al perfeccionamiento profesional del profesorado, pero son su actitud y sus expectativas ante lo que la reforma del sistema educativo puede suponer, los factores que decidirán el éxito o el fracaso de la misma.

\section{REFORMA Y TRANSVERSALIDAD}

Nos hemos referido en otras ocasiones ${ }^{1}$ a las condiciones necesarias y a los obstáculos que deben considerarse fundamentales en el logro de los objetivos de la reforma en relación al profesorado.

En esta ocasión nos referimos a las condiciones necesarias que deben tenerse en cuenta si pretendemos abordar la acción pedagógica en su plenitud y con el grado de transversalidad necesario para que la triple dimensión de los contenidos de aprendizaje sea atendida de forma satisfactoria.

En la aportación que en esta misma revista desarrolla Josep M. Puig, éste plantea que en el currículum de educación moral como mínimo deben considerarse actividades específicas transversales y sistemáticas de educación moral, actividades y proyectos de centro que potencien la participación democrática en la vida colectiva de la institución educativa y actividades propedéuticas para la participación social.

Estos aspectos son un conjunto de indicadores de lo que puede caracterizar un currículum específico de dimensión claramente transversal y que afecta no sólo a las situaciones de educación formal sino también a aquellas situaciones de educación no formal que tienen lugar en el marco de los centros educativos y que dotan de sentido a la vida colectiva y diaria que en ellos se desarrolla.

Puesto que las actividades que proponemos no están contempladas como áreas curriculares específicas y deben ser abordadas de forma multidisciplinar por el conjunto de todo el profesorado, se nos presentan en su conjunto como un claro ejemplo de transversalidad que no debe ni puede confundirse con el conjunto de contenidos de aprendizaje relativos a las actitudes, valores y normas de las diferentes áreas curriculares.

Nuestra propuesta curricular transversal no sólo afecta a contenidos actitudinales, valorativos y normativos, sino que también incluye, y forma destacada, contenidos procedimentales y contenidos relativos a hechos, conceptos y sistemas conceptuales. Podría ser objeto de un área curricular específica con un horario escolar específico, pero no es imposible abordarla de forma multidisciplinar si el nivel de trabajo en equipo del profesorado es notable y si está integrada en el proyecto educativo de centro a través de un proceso participativo y responsable.

El carácter transversal y la consideración no sólo formal sino también no formal e informal de los ámbitos en los que pueden desarrollarse actividades de educación moral, participación democrática en la vida colectiva del centro y preparación para la vida social son condiciones que exigen una reflexión profunda sobre el estilo pedagógico de los centros y las actitudes del profesorado.

\section{LAS ACTITUDES DEL PROFESORADO}

Cuando nos referimos a las actitudes del profesorado no sólo queremos senalar las actitudes en el aula ante los conflictos de valor, aspecto que es desarro- 
llado en esta misma revista por Jaume Trilla, sino que también nos referimos a aquellas actitudes en torno al trabajo en equipo, la aceptación crítica del otro y de sus opiniones o puntos de vista diferentes o contrarios a los nuestros, la participación en un proyecto educativo de centro no sólo en su fase de elaboración sino en los diferentes momentos de su puesta en práctica diaria y la búsqueda del consenso a través del diálogo, que haga posible poder hablar de todo aquello en lo que no estamos de acuerdo en nuestra forma de hacer pedagógica, convencidos de que aproximando perspectivas es la mejor forma de proceder para resolver los conflictos.

Estas actitudes son convenientes para el logro de un ambiente profesional que facilite el trabajo en equipo y haga posible plantearse objetivos pedagógicos de claro carácter transversal y que afecte a las dimensiones más basales de nuestra acción pedagógica en las aulas, como puede ser el ámbito de la educación en valores y de la educación moral.

En párrafos anteriores afirmábamos que existen formas de abordar las innovaciones pedagógicas más óptimas que otras, y ahora hemos formulado algunas actitudes y disposiciones más convenientes, necesarias, a nuestro juicio, para abordar nuestra propuesta curricular de educación en valores y educación moral en su sentido más completo.

En otras ocasiones hemos insistido en la necesidad de sistematizar las actividades de educación moral en su sentido más completo.

En otras ocasiones hemos insistido en la necesidad de sistematizar las actividades de educación moral y en evitar a toda costa que éstas queden relegadas a un simple conjunto de actividades sin orden ni concierto, ocasionales, y para cubrir espacios horarios vacíos o incluso ausencias o bajas del profesorado tutor de cada aula. Es importante abordar nuestra propuesta curricular con el mayor rigor y ordenación posible, y para ello ofrecemos en las diferentes colaboraciones que constituyen este número una serie de estrategias, técnicas o metodologías a seguir en la realización de las diferentes actividades.

Hemos dispuesto diferentes ejemplos de actividades según las diversas técnicas o estrategias consideradas, con la intención de ofrecer una serie de ejemplos que sólo pueden ser útiles si se someten a un proceso de revisión y se integran en la realidad de cada centro y aula, teniendo en cuenta el contexto socio-cultural del mismo y las diferencias y conflictos que en él puedan plantearse o convenga plantear porque están presentes en contextos sociales más amplios.

Es así que el peor uso que podía hacerse de nuestro trabajo sería aplicarlo sin más, sin reflexión ni integración en el proyecto educativo de centro y, de forma especial, en el conjunto de actividades que en cada aula el profesorado desarrolla y que, por supuesto, afectan a diferentes áreas curriculares.

Esta insistencia en la sistematicidad no debe suponer sólo el conocimiento de las diferentes estrategias y técnicas, sino que debe además suponer la integración de las diferentes actividades en el sistema que conforma el conjunto de la actividad pedagógica en el aula y en el centro.

Los materiales curriculares que nuestro grupo de investigación está elaborando en su fase final ${ }^{2}$ deben entenderse como propuestas que pueden no sólo ser aplicados sino sobre todo ser considerados como ejemplos para su recreación en el contexto de cada aula y centro específico:

Por todo lo expuesto anteriormente, creemos conveniente abordar nuestra propuesta curricular y, en general, toda innovación pedagógica a partir de un modelo circular que relacione sistemáticamente la propuesta teórica de la que 
se trate con la realidad pedagógica en la que debe realizarse continua revisión, desde la práctica pedagógica y por los que van a ser los autores directos de su puesta en escena. Pero, además, este diálogo no debe hacerse suponiendo que partimos de cero, que el estado inicial es ajeno o incluso opuesto a lo que perseguimos. Probablemente el estado incial es más óptimo de lo que podemos suponer y no conviene en modo alguno prescindir de él.

De la misma forma que han existido maestros piagetianos mucho antes de que Piaget formulara su teoría y pudieran conocerse sus implicaciones pedagógicas, es posible que ante una innovación pedagógica de interés existan realidades pedagógicas, aulas y centros, que están organizados y funcionan como si tal innovación fuera ya conocida y practicada desde hace tiempo. Las fuentes del conocimiento pedagógico no sólo se basan en el desarrollo teórico y de la investigación pedagógica de carácter científico. Existen fuentes de conocimiento ordinario que no sólo son útiles sino que en ocasiones son los que han hecho posible progresar en el aula día a día a pesar de su escasa o nula sistematicidad y contraste.

\section{ALGUNAS CONSIDERACIONES ANTE LAS INNOVACIONES PEDAGÓGICAS}

La primera consideración necesaria ante toda la innovación es abordarla reduciendo los niveles de ansiedad y preocupación ante lo nuevo. Una adecuada autoobservación de lo que hacemos en las aulas puede ser un primer paso importante para reconocer si lo que se nos presenta como nuevo es realmente ajeno a lo que habitualmente hacemos. En nuestro caso, en la propuesta curricular que hacemos, es probable que no sólo no sea ajeno sino que esté presente desde siempre, aunque quizás de forma incompleta, inconsciente e inintencional.

Si este ejercicio de autoobservación es compartido por el resto del profesorado del mismo nivel o etapa, los niveles de ansiedad o preocupación pueden decrecer notablemente. Es difícil concebir un centro educativo en el que la preocupación por los valores y la moral no esté presente de alguna forma en la práctica pedagógica habitual del aula y de la institución.

Por el contrario, es fácil imaginar y recordar que en numerosas ocasiones después de esta primera fase, nuestro estado de atención ha decrecido o incluso olvidado que toda innovación teóricamente rigurosa y metodológicamente bien estructurada puede ser útil y debe ser aprovechada para reducir los niveles de asistematicidad, inintencionalidad y falta de secuenciación que la práctica sin teoría conlleva frecuentemente.

Por ello, es necesaria una segunda consideración en la forma de abordar las innovaciones pedagógicas. Consiste en analizar en profundidad el marco teórico en el que tal innovación surge, en nuestro caso la bibliografía sobre el tema es suficiente y el marco teórico de nuestra propuesta curricular en concreto puede consultarse sin dificultad ${ }^{3}$.

Este análisis conviene realizarlo desde la práctica pedagógica, pero con voluntad de superar lo cotidiano, integrándolo en el modelo teórico que defiende buscando los puntos de coincidencia y discrepancia y detectando las lagunas que a través de las diversas lecturas puedan constatarse. La reflexión así realizada y la contrastación con la práctica habitual puede ayudarnos a comprender que no se trata de añadir una nueva área o «asignatura» ni de incrementar el 
tiempo de trabajo lectivo del profesorado y de los alumnos. Se trata de reordenar lo que ya hacemos, simplificando algunas de nuestras actividades en el aula e implementando nuestra acción en torno a los valores y lo moral al tratar contenidos de diferentes áreas. Y ello es posible desde una perspectiva que contemple especialmente lo valorativo, el juicio basado en criterios personales, el diálogo, el uso de la razón para el análisis de la realidad, la empatía y el desarrollo de competencias en el alumnado que hagan posible manifestar conductas y acciones coherentes con el juicio elaborado, así como dedicando el tiempo necesario al conocimiento de aquellos hechos y conceptos que de carácter natural, social y ético sean necesarios para el completo desarrollo de nuestra propuesta curricular.

La tercera consideración consiste en el estudio de las diferentes estrategias y técnicas, algunas específicas y otra comunes a otras áreas curriculares, que pueden ser útiles para el trabajo en el aula y el centro. Como se puede comprobar, la mayoría de técnicas y estrategias son metodologías afines o coincidentes con las que ya están presentes en el proceder de un amplio sector del profesorado; sin embargo, el conocimiento en detalle de los protocolos y condiciones para su correcta aplicación facilita el trabajo posterior y permite actuar con mayor seguridad, sobre todo cuando se trata de diseñar nuevas actividades por el profesorado de cada centro, objetivo éste, a nuestro juicio, fundamental como ya hemos señalado.

Es posible que a través de las tres consideraciones que presentamos y especialmente a través de la comparación de la primera, autoobservación y diagnóstico de lo que ya hacemos con la segunda y la tercera de carácter teórico y metodológico estemos en condiciones de abordar los diferentes niveles de concreción curricular, especialmente el segundo y tercero, con seguridad y eficacia.

Nuestra intención al ofrecer los materiales que ahora presentamos es contribuir a que un reto tan importante como el de la reforma del sistema educativo pueda realizarse desde la práctica actual y mediante la reflexión y optimización que todo proceso de creación y renovación pedagógica debe suponer.

No creemos que sean los materiales, ni el aprendizaje de nuevas técnicas y estrategias la garantía del necesario proceso de reforma en el que nos encontramos. Creemos que la reforma debe redefinir nuestra función pedagógica y cambiar nuestra forma de aproximarnos a la tarea diaria en el centro y en el aula.

\section{Notas}

1 Martínez, M. (1991) «Rol del educador y de la escuela en el ẢMBITO DE LA EDUCACIÓN moral«, Martínez, M., Puig. J. M. (Coord.) (1991) La educación moral. Perspectiva de futuro y técnicas de trabajo. Barcelona: Graó. pp. 19-29.

Martínez, M. (1992) «Aprendre i ensenyar», Martínez, M., Monereo, C., Gallifa, J i Mateo, J. (1992). Educar és un procés. Barcelona: Editorial Cruilla. pp 13-61.

2 Los materiales curriculares que estamos elaborando constituyen nuestro trabajo fundamental a lo largo de los últimos tres años y han sido posibles gracias a las ayudas recibidas del CIDE de la Secretaria de Estado de Educación y el ICE de la Universidad de Barcelona a la que pertenecemos.

3 Puig, J. M. i Martínez, M. (1989) Educación Moral y Democracia. Barcelona: Laertes. Buxarrais i altres (1990) Etica i escola: El tractament pedagògic de la diferència. Barcelona: Ed 62. VV.AA. (1991) L'interculturalisme en el currículum. El Racisme. Barcelona: Rosa Sensat. Martínez, M. i Puig, J.M. (1991). La Educación Moral. Perspectivas de futuro y técnicas de trabajo. Barcelona: Graò (1992) Cuadernos de Pedagogía. 


\section{Educación en valores y Educación Moral:}

Un ámbito de diseño y construcción

curricular para el profesorado

Miquel Martínez Martín

CL\&E, 1992, 15, pp. 13-18

Resumen: Se analiza la noción de transversalidad en el marco de la Reforma del Sistema Educativo y su relación con el carácter transversal de las actividades de Educación Moral y Educación en valores. Se consideran algunas de las actitudes del profesorado que hacen posible plantearse objetivos pedagógicos no específicos de área y se analizan aquellas consideraciones ante las innovaciones pedagógicas que pueden contribuir a afrontarlas de forma satisfactoria.

Datos sobre el autor: Miquel Martínez Martín es Catedrático de Teoría de la Educación de la Universidad de Barcelona y miembro del Grupo de Investigación en Educación Moral (G.R.E.M.).

Dirección: Departamento de Teoría e Historia de la Educación, Facultad de Pedagogía, Universidad de Barcelona, Baldiri Reixac, s/n 08028 Barcelona. Tel (93) 3333466.

(C) De todos los artículos deberá solicitarse por escrito autorización de CL\&E y de los autores para el uso en forma facsímil, fotocopia o cualquier otro medio de reproducción impresa. CL\&E se reserva el derecho de interponer las acciones legales necesarias en aquellos casos en que se contravenga la ley de derechos de autor. 